\title{
Effectiveness of antibiotics
}

\section{for acute sinusitis in real-life medical practice}

\section{Patrick Blin, ${ }^{1,2}$ Sylvie Blazejewski, 1,2,3,4 Séverine Lignot, ${ }^{1,2}$}

Régis Lassalle, 1,2 Marie-Agnès Bernard, 1,2 Delphine Jayles, ${ }^{1,2}$

Hélène Théophile, ${ }^{4}$ Jacques Bénichou, ${ }^{4,5}$ Jean-Louis Demeaux, ${ }^{1}$

David Ebbo, ${ }^{6}$ Jacques Franck, ${ }^{7}$ Yola Moride, ${ }^{1,8}$

Dominique Peyramond, ${ }^{9}$ Bernard Rouveix, ${ }^{10}$ Miriam Sturkenboom, ${ }^{11}$

Paul Gehanno, ${ }^{12 *}$ Cécile Droz ${ }^{1,2,4}$ \& Nicholas Moore, ${ }^{1,2,3,4}$

'Université Bordeaux 2, Bordeaux, France, ${ }^{2}$ INSERM CIC 0005, Bordeaux, France, ${ }^{3} \mathrm{CHU}$ de Bordeaux, Bordeaux, France, ${ }^{4}$ INSERM U657, Bordeaux, France, ${ }^{5} \mathrm{CHU}$ de Rouen, Rouen, France, ${ }^{6}$ Groupe Hospitalier Paris-Saint-Joseph, Paris, France, ${ }^{7}$ General Practitioner, Rouen, France, ${ }^{8}$ Université de Montréal, Montréal, Canada, ${ }^{9} \mathrm{Hôpital}$ de la Croix Rousse, Lyon, France, ${ }^{10} \mathrm{Hôpital} \mathrm{Cochin,} \mathrm{Paris,} \mathrm{France,}{ }^{11}$ Erasmus Medical Center, Rotterdam, The Netherlands, and ${ }^{12}$ Hôpital Bichat, Paris, France
Correspondence

Dr Patrick Blin, Département de

Pharmacologie, Université Victor Segalen Bordeaux 2, 146 Rue Léo Saignat, 33076 Bordeaux cedex, France.

Tel.: + 33 (0) 557571560

Fax: +33 (0) 557574671

E-mail:

patrick.blin@pharmaco.u-bordeaux2.fr

*Died 6 January 2007

Keywords

acute sunisitis, antibiotics, effectiveness, pharmacoepidemiology, cohort study

\section{Received}

29 October 2009

Accepted

13 May 2010

\section{WHAT IS ALREADY KNOWN ABOUT} THIS SUBJECT

- Determining bacterial aetiology of acute sinusitis is difficult without employing invasive procedures.

- Most episodes of acute sinusitis resolve spontaneously.

- Antibiotics have demonstrated efficacy for the treatment of acute bacterial sinusitis in clinical trials yet little is known of their effectiveness in real-life treatment settings.

\section{WHAT THIS STUDY ADDS}

- Most cases of untreated acute sinusitis resolved spontaneously. Antibiotics were more effective when given within the first 10 days of treatment. This had no effect on later recurrence.

- Patients with poor oro-dental condition or recent antibiotic use may derive the most benefit from an antibiotic prescription and this should be considered by prescribers. - The antibiotics used were found to be equally effective.

- Existing recommendations to identify acute sinusitis with high probability of bacterial origin, such as the French recommendations, fever or duration of symptoms fail to identify patients in whom antibiotics are more effective.

\section{AIMS}

To assess the effectiveness of antibiotics in acute bacterial sinusitis.

\section{METHODS}

This was a prospective cohort study with 2 months follow-up of 5640 patients with acute sinusitis included by a random sample from 1174 GPs and 120 ENT specialists. Main outcomes were short-term initial success, defined as the absence of prescription of (another) antibiotic or sinus lavage within 10 days, and lack of recurrence between the $11^{\text {th }}$ and $60^{\text {th }}$ day, after initial success.

\section{RESULTS}

Initial success was found in $88.7 \%$ (95\% Cl 85.1, 91.4\%) of patients without antibiotic prescription at inclusion and 96.2\% (95\% Cl 95.7, $96.7 \%)$ of patients prescribed antibiotics. The 10 day adjusted hazard ratio $(\mathrm{HR})$ for treatment failure (new antibiotic prescription or sinus drainage) with initial antibiotics compared with no antibiotics was 0.30 $(95 \% \mathrm{Cl} 0.21,0.42)$ with no difference between antibiotics. Antibiotics were more effective in patients with poor oro-dental condition (HR $0.04,95 \% \mathrm{Cl} 0.01,0.20)$ and in patients who had already used antibiotics during the previous 2 months (HR $0.09,95 \% \mathrm{Cl} 0.03,0.28$ ). For patients without failure at 10 days, recurrence between the $11^{\text {th }}$ and $60^{\text {th }}$ day was similar whether or not they had initially been prescribed an antibiotic, $94.1 \%(95 \% \mathrm{Cl} 93.4,94.7 \%)$ and $93.4 \%(95 \% \mathrm{Cl} 90.3$, $95.5 \%)$, respectively.

\section{CONCLUSION}

Most acute sinusitis cases not prescribed antibiotics resolve spontaneously. Antibiotics reduced by 3.3-fold the risk of failure within 10 days, without impact on later recurrence. The greatest benefit of antibiotics was found for patients with poor oro-dental condition or with antibiotic use within the previous 2 months. 


\section{Introduction}

Acute sinusitis (AS), also called acute rhinosinusitis, is an inflammation of one or more of the paranasal sinuses that lasts less than 4 weeks [1]. It is classified as either acute bacterial sinusitis (ABS) or viral sinusitis [2]. It is one of the most common diseases seen by primary care physicians [3, 4]. In Europe ABS represents $1 \%$ to $2 \%$ of adult patient visits $[5,6]$. Diagnosis of uncomplicated ABS relies mainly on history, symptoms and clinical examination $[2,3,7,8]$. Sinus imaging is rarely performed at initial assessment and furthermore has been shown to distinguish poorly between bacterial and viral aetiologies [7,9]. Sinus puncture for bacterial examination, considered as the gold standard for bacterial diagnosis, is reserved for patients at high risk of complications or antibiotic resistance, or for research purposes [3, 9]. Guidelines and national recommendations have been proposed for the use of a combination of clinical symptoms to identify AS with a high probability of bacterial origin $[7,8,10,11]$. As a consequence, treatment of non-complicated presumed ABS in real-life clinical practice is empirical and often includes a probabilistic antibiotic treatment covering bacteria commonly associated with this condition, Streptococcus pneumoniae, Haemophilus influenzae and Moraxella catarrhalis [2, 3, 8, 12, 13].

Efficacy of antibiotics in ABS has been widely demonstrated in randomized clinical trials [12, 14-16]. However, the management of acute sinusitis in a real-life setting is far from the experimental conditions found in clinical trials $[4,17]$. For instance, in real-life management of AS, physicians are not selected nor specifically trained, patients are not excluded for other medical and non-medical conditions, sinus imaging and bacterial sampling are not used to confirm the diagnosis, management of care (drug dosage and duration, surveillance and follow-up) are not fixed by a protocol. Furthermore, in current medical practice, outcome is more often defined by a subsequent visit for lack of improvement or worsening resulting in change of prescription or in sinus drainage, rather than by cure or improvement which might be thought implicit when there is no further prescriber intervention. Such differences could impact on treatment effectiveness [17-19]. Therefore the objective of the current study was to assess in real-life clinical practice the effectiveness of antibiotics in AS in a large prospective cohort of out-patients recruited by general practitioners (GPs) and ear, nose and throat (ENT) specialists.

\section{Methods}

An observational prospective cohort study was designed to include 6000 patients with AS by a national random sample of GPs and ENT specialists during two periods, March-June 2005 and September-December 2005, to account for possible seasonal differences in AS. Physicians were identified using the French telephone directory database (France Telecom) and recruited by postal mail.

Each participating physician was asked to register prospectively all the patients consulting for an episode of AS diagnosed according to their own usual criteria, up to a maximum of 20 patients or for 4 weeks. They were asked to include in the AS cohort the first five patients of the registry with the following criteria: diagnosis of AS, age 18 years or over, accepting to participate in the study, without history of AS over the 2 preceding months (in order to not include failures of previous treatments), who could be followed-up over the 2 subsequent months, presenting no severe disease that would be life threatening within the 3 subsequent months or require the use of antibiotics during that period, and not participating in a controlled clinical trial. The initial therapeutic choice by the recruiting physician was indifferent. During the SeptemberDecember 2005 inclusion period, the number of patients included by each physician was increased from five to six, in order to reach the total planned number of patients. Patients were followed-up for 2 months with evaluations at 10 days and 2 months after inclusion, by telephone contact or upon the occasion of a visit to the physician for any reason. There was no systematic visit planned in the protocol. In parallel, patients had to fill out a diary over the 10 days following inclusion, which was returned by mail to the investigation centre.

In order to avoid selection and information bias, physicians knew of the general objective of evaluation of the effectiveness of initial treatment of AS but were not informed of the specific study objective of antibiotic effectiveness or the name of the study sponsor before the end of the study. This was approved by the National professional ethics committee.

\section{Data collected}

Data collected by the physician in the registry were visit date, gender and age of the patient, AS localization, therapeutic class of drugs prescribed for AS, patients included or not in the cohort and reason(s) for non-inclusion.

At inclusion in the cohort, the physician collected the patient's initials, inclusion and non-inclusion criteria, visit date, demographic characteristics, smoking status, presence of air conditioning in the living or working environment, regular contact with children under 2 years of age, concomitant diseases, history of AS, history of infections over the past 2 weeks, antibiotic use over the past 2 months, self-medication before inclusion, fever $\left(\geq 38.5^{\circ} \mathrm{C}\right)$, symptoms and localization of current AS, drugs prescribed at inclusion, sinus irrigation performed at inclusion, ENT specialist referral (for GPs), sinus imaging, bacterial examination and hospitalization for AS.

At day 10 , the physician collected the date, AS evolution, premature discontinuation of initial strategy with reasons and date, possible antibiotic prescription after inclusion and date of prescription, sinus irrigation and date 
of procedure, ENT specialist referral (for GPs), sinus imaging, bacterial examination and hospitalization for AS after inclusion.

At day 60 , the physician collected the date, change in signs and symptoms of AS since day 10, antibiotic prescription since day 10 and date of prescription, sinus irrigation and date of procedure, ENT visit, sinus imaging, bacterial examination and hospitalization for AS since day 10 .

In the diary, patients were required to report the following data every day from inclusion to day 10: sinus pain, temperature, purulent rhinorrhoea, nasal obstruction, headache, drugs used for AS, visit to GP or ENT specialist or emergency room, sinus imaging, sinus irrigation, bacterial examination and hospitalization related to AS.

\section{AS diagnosis validation and effectiveness criteria}

Validation of physician AS diagnosis and classification as high probability of bacterial origin were done by checking whether the symptoms reported by the physician met the French recommendations for the diagnosis of ABS, as defined by a national study group of infectious disease specialists $[7,10,11]$. Three criteria were considered (clinical signs, localization and characteristics of pain) and adapted to each type of sinusitis. For maxillary sinusitis this was unilateral purulent anterior rhinorrhoea and/or posterior rhinorrhoea, sub-orbital pain, unilateral pain and/or pulsatile pain and/or with climax at the end of the day or at night. For ethmoido-frontal sinusitis it was posterior rhinorrhoea and/or purulent uni- or bilateral anterior rhinorrh-ea and/or palpebral oedema, frontal pain, pulsatile pain and/or with climax at the end of the day or at night. For sphenoidal sinusitis it was posterior rhinorrhoea, vertex and/or retro-orbital pain, uni- and/or bilateral pain and/or pulsatile pain and/or with climax at the end of the day or at night. Pansinusitis was defined as two or more of the above localizations.

Effectiveness of antibiotics was determined by the absence of treatment failure within the first 10 days after inclusion (initial treatment success) and the absence of recurrence of sinusitis between the $11^{\text {th }}$ and the $60^{\text {th }}$ days after inclusion in those patients who were judged successful at day 10 . Failure and recurrence were defined by sinus drainage or new antibiotic prescription (switch or initiation). Recurrence could be due to relapse with the same bacterial strain or re-infection with a new strain but it was not possible to distinguish between the two since systematic bacterial sampling is not commonly done in current clinical practice.

\section{Statistical analyses}

Descriptive analyses were weighted to take into account the true proportion of GPs and ENT specialists, using national physician statistics [20], and the physicians' monthly AS activity assessed using the study registries [21], in order to avoid over-representation in the study of
ENT and physicians with low activity. Descriptive results are presented as percentage or mean and standard deviation (SD). Student's $t$-test and the Chi square test were used to compare initial characteristics between groups prescribed or not prescribed antibiotics, with a $P$ value below 0.05 as the level of statistical significance.

The effectiveness criteria, failure or recurrence, were described using the Kaplan-Meier method for patients with and without antibiotics. Prognostic factors of failure within the first 10 days were assessed using Cox proportional-hazards regression, using the following predictors: physician type (GP or ENT specialist), gender and age of the patient, smoking status, history of allergic sinusitis or asthma, poor oro-dental condition, history of AS over the past 12 months, antibiotic use over the past 2 months, viral infection over the past 2 weeks, fever $\left(\geq 38.5^{\circ} \mathrm{C}\right)$, self medication before the inclusion physician visit (nasal preparations or decongestants, analgesics, non-steroidal anti-inflammatory drugs (NSAIDs), AS localization, delay between symptom onset and visit, number of ABS diagnostic criteria according to the current French recommendations and treatment prescribed at inclusion (nasal preparations or decongestants, cough and cold preparations, antibiotics, analgesics, NSAIDs, corticosteroids). Subgroup analyses were performed using adjustments of the preceding model in order to evaluate result stability and interactions. Prognostic factors of recurrence between the $11^{\text {th }}$ and the $60^{\text {th }}$ days were assessed for patients without failure within the first 10 days using the Cox proportionalhazards regression model with the same procedure as above. Cox proportional-hazards regression results are presented as a hazard ratio (HR) with $95 \%$ confidence intervals $(95 \% \mathrm{Cl})$. A general linear model was used to compare duration of symptoms within the first 10 days between antibiotic prescribed and not-prescribed groups with adjustment on all variables included in the failure Cox model.

Five physicians were excluded from the analysis because they had not filled out their registry. Patients were excluded in the case of AS localization not reported (24 patients), missing information for effectiveness criteria (31 patients) and hospitalization at inclusion because of uncertainty as to the initial treatment prescribed during hospitalization (10 patients).

\section{Results}

During the two inclusion phases (March-June 2005 and September-December 2005), 5640 patients were included in the cohort, 5200 by 1174 GPs and 440 by 120 ENT specialists. The diary was completed by $89.1 \%$ of the patients. The mean number of patients with an AS diagnosis per month was 10.8 (SD 9.8) for GPs and 10.4 (SD 11.3) for ENT specialists according to the study registries. By extrapola- 
tion to all French GPs and ENT specialists it appears that $96.7 \%$ of patients with AS are seen by a GP.

\section{Patients}

Taking into account the proportion of GPs and ENT specialists in France participating in the study and their activity regarding AS, the weighted analysis showed that patients with AS had a mean age of 42.5 (SD 14.3) years, 39.9\% were men and $29.8 \%$ were current smokers. Co-morbidities declared for more than $1 \%$ of patients were allergic rhinitis (19.4\%), poor oro-dental condition (7.6\%), asthma (7.0\%), nasal polyposis $(4.4 \%)$, diabetes $(2.7 \%)$, respiratory insufficiency $(1.4 \%)$ and history of facial trauma (1.2\%). One third of the patients $(33.2 \%)$ had a history of AS during the past 12 months and $50.7 \%$ had had a viral infection other than AS over the 2 weeks preceding inclusion, primarily rhinopharyngitis (40.9\%) and flu (9.4\%). An antibiotic prescription over the past 2 months was declared for $9.0 \%$ of patients for various infections: bronchitis (31.6\%), tonsillitis (18.9\%), rhinopharingitis without AS (13.7\%), urinary infection (9.5\%), dental infection (6.9\%), otitis (2.9\%), skin infection $(2.1 \%)$, pneumopathy $(1.7 \%)$, flu $(1.7 \%)$, other infection (5.1\%) and not specified (5.9\%). Self-medication used before the physician visit was mainly painkillers (analgesics $51.1 \%$ or NSAIDs $10.2 \%$ ) and nasal preparations or decongestants (34.6\%). Most patients consulted the study physician within the first week after symptom onset, 31.2\% within the first 2 days and $50.6 \%$ between days 3 and 7 .

Sinusitis localization was maxillary for $48.0 \%$ of patients, ethmoido-frontal for $28.5 \%$, sphenoid for $0.7 \%$ and pansinusitis for $22.8 \%$. Fever was reported at inclusion for $43.2 \%$ of the patients. From the analysis of symptoms reported at inclusion, $85.3 \%$ had at least two diagnostic criteria for high probability of $A B S$.

Drugs prescribed at inclusion were antibiotics $(92.4 \%$ of patients), nasal preparations and decongestants $(81.1 \%)$, analgesics $(50.2 \%)$, corticosteroids $(48.8 \%)$, expectorants and cough suppressants (44.8\%) and NSAIDs (18.0\%). Antibiotics prescribed to more than $1 \%$ of the patients were cefpodoxime (18.3\%), co-amoxiclav (17.7\%), telithromycin $(11.9 \%)$, cefuroxime $(10.4 \%)$, pristinamycin (9.1\%) moxifloxacin (8.0\%), levofloxacin (3.3\%), amoxicillin $(2.8 \%)$, cefotiam (2.5\%), clarithromycin (2.4\%), ciprofloxacin (1.3\%) and cefixime (1.2\%). Mean antibiotic treatment duration declared by patients in their diary varied from 5.4 (SD 1.7) days for telithromycin to 8.2 (SD 1.8) days for co-amoxiclav and was at least 5 days for more than $90 \%$ of the patients. There was no difference in patient or sinusitis characteristics between the different antibiotics considered.

\section{Patients prescribed antibiotics}

In the group prescribed antibiotics, there were significantly more men, patients without allergic rhinitis comorbidity, patients visiting between 3 and 7 days after symptom onset, patients with three ABS diagnosis criteria and patients with one of the following conditions: poor orodental condition, history of AS over the past 12 months, viral infection over the past 2 weeks, analgesic self medication and fever (Table 1). Other prescriptions for these patients included significantly more corticosteroids and fewer NSAIDs, nasal preparations and decongestants (Table 1). Mean ( $\pm S D$ ) duration of treatment with other prescribed drugs was significantly shorter for patients prescribed antibiotics: analgesics $4.2 \pm 2.3$ days vs. $5.1 \pm 3.0$ days, $P<0.001$, NSAIDs $4.6 \pm 2.0$ days vs. $5.2 \pm 2.2$ days, $P=$ 0.01 and corticosteroids $5.0 \pm 1.9$ days vs. $5.5 \pm 2.1$ day, $P=0.02$.

\section{Initial treatment success}

Initial treatment was successful in $88.7 \%(95 \% \mathrm{Cl} 85.1$, $91.4 \%)$ of the patients not prescribed antibiotics at inclusion and $96.2 \%(95 \% \mathrm{Cl} 95.7,96.7 \%)$ of the patients prescribed an antibiotic (Figure 1). Failures were identified by a new antibiotic prescription in $97.3 \%$ of the failures in the group prescribed antibiotics and in all failure patients in the group not prescribed antibiotics. No serious complication was reported during the study. Cox proportionalhazards model found three significant risk factors for treatment failure, all patients considered (Table 2): respiratory insufficiency (HR $2.72,95 \% \mathrm{Cl} 1.40,5.27)$, antibiotic use in the 2 months prior to inclusion (HR 1.60, 95\% Cl 1.11, 2.30), female gender ( $\mathrm{HR} 1.32,95 \% \mathrm{Cl} 1.00,1.75)$ and one significant protective factor: antibiotic prescription ( $H R$, $0.30,95 \% \mathrm{Cl} 0.21,0.42)$. A model including the different antibiotics found no independent effect of individual drugs. (data not shown) The HR to be cured by an antibiotic was $1.09(95 \% \mathrm{Cl} 1.07,1.10)$ and represents a number needed to treat (NNT) to avoid one failure of 13 patients $(95 \% \mathrm{Cl} 11,14)$. Other treatments (analgesic, NSAIDs, corticosteroid, nasal preparations and decongestants, mucolytics) had no impact on risk of failure. If antibiotic use over the 2 previous months was removed from the model, viral infection over the past 2 weeks was still not significant $(P=0.64)$.

The protective effect of antibiotic treatment was found in all subgroups studied (Figure 2). None of the subgroups had a HR point estimate above the upper bound of the confidence interval of the overall HR (i.e. less protective effect). The ENT HR was not calculable because no failure was observed for the 45 ENT patients without initial antibiotics. Antibiotics were even more effective in patients with poor oro-dental condition ( $\mathrm{HR} 0.04,95 \% \mathrm{Cl} 0.01,0.20$ ) and in patients who had already used antibiotics during the previous 2 months (HR $0.09,95 \% \mathrm{Cl} 0.03,0.28)$. The protective effect seemed also to be better for men than for women ( $\mathrm{HR} 0.19,95 \% \mathrm{Cl} 0.10$ to 0.33 , vs. $0.38 ; 95 \% \mathrm{Cl} 0.24$, $0.59)$, though there was overlap in the confidence intervals

Mean ( \pm SD) duration of symptoms in the group prescribed antibiotics compared with the group not prescribed antibiotics was significantly shorter for purulent rhinorrhoea ( $4.4 \pm 2.5$ days vs. $5.1 \pm 3.6$ days, $P<0.001)$, 


\section{Table 1}

Characteristics of patients at inclusion according to antibiotic prescription (weighted analysis)

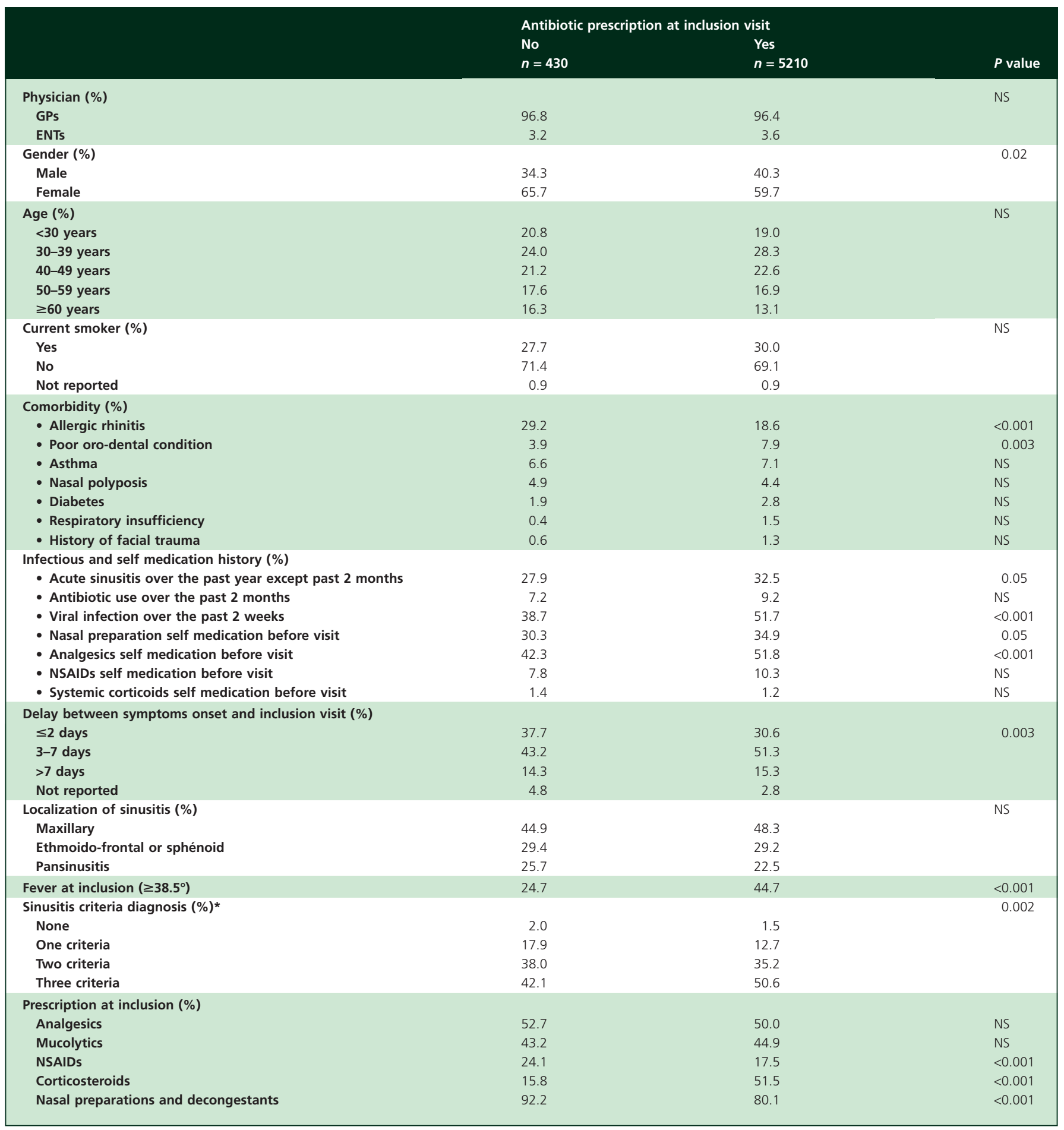

*According to the French recommendations for the diagnosis of ABS defined by a national study group of infectious disease specialists [10]. NS, non significant.

nasal obstruction $(4.1 \pm 2.6$ days vs. $5.1 \pm 3.3$ days, $P<0.001)$, headache $(3.6 \pm 2.4$ days vs. $4.0 \pm 2.8$ days, $P=0.003)$, and not statistically different for sinus pain (3.9 \pm 2.2 days vs. $4.1 \pm 2.7$ days, $P=0.06)$ and fever $(2.3 \pm 1.4$ days vs. $2.3 \pm 1.5$ days, $P=0.69)$.

\section{Recurrence}

Absence of initial failure or of recurrence at day 60 was found in $90.6 \%(95 \% \mathrm{Cl} 89.8,91.4 \%)$ of patients with an antibiotic at inclusion and $83.0 \%$ (95\% Cl 79.0, 86.3\%) of the patients without antibiotic prescription (Figure 1). For 


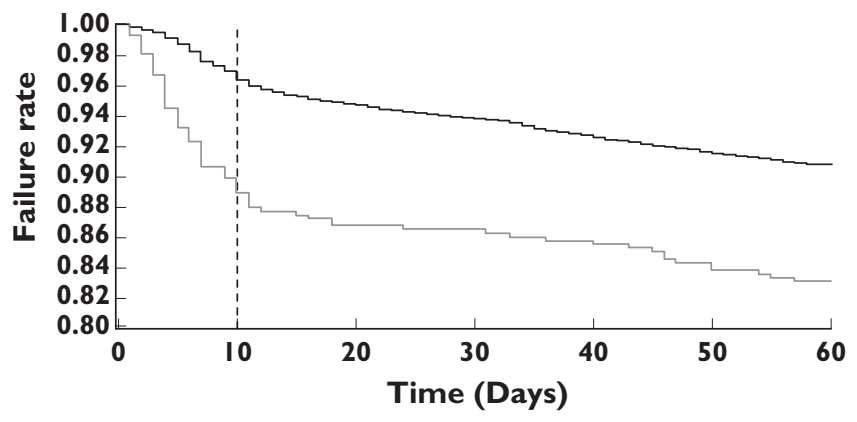

\section{Figure 1}

Time to failure or recurrence between days 0 and day 60 according to antibiotic prescription. No initial antibiotic prescription (- - ; Initial antibiotic prescription $(-)$

patients with initial success within the first 10 days, absence of recurrence was similar whether they initially had an antibiotic prescribed or not $(94.1 \%, 95 \% \mathrm{Cl} 93.4$, $94.7 \%$ and $93.4 \%, 95 \% \mathrm{Cl} 90.3,95.5 \%$, respectively. For these patients with initial success, predictive factors for recurrence between 11 and 60 days were history of facial trauma (HR 2.14,95\% Cl 1.00,4.55), poor oro-dental condition (HR $1.81,95 \% \mathrm{Cl} 1.29,2.55)$, history of sinusitis over the past 12 months (HR 1.59, 95\% Cl 1.27, 1.99). None of the initial treatment options had a positive or negative impact on the risk of recurrence beyond the first 10 days.

\section{Discussion}

This is the first large prospective ad hoc cohort study to address antibiotic effectiveness in AS in real-life medical practice. Patients to whom the physician chose to prescribe antibiotics were less prone to treatment failure within the first 10 days. Though there were significant differences at inclusion between patients prescribed antibiotics and those not prescribed antibiotics, multivariate analysis adjusted on differences at inclusion found that initial antibiotic prescription reduced 3.3-fold the risk of failure within 10 days, but had no impact on later recurrence. Furthermore, patients prescribed antibiotics had shorter duration of symptoms and treatment by symptomatic drugs.

One limitation of this study was the use of a care criterion to define failure and not a clinical assessment. However, this pragmatic criterion reflects a real-life definition of effectiveness where patients go back to their physician only in case of worsening or no improvement after a few days. Patients who do not have a new antibiotic prescription or sinus drainage can be considered cured of the acute event. A similar definition of failure (new antibiotic prescription) has been used as a criterion in claims database AS studies $[18,19]$. In these, the success rate at 28 days was $90.4 \%$ with any antibiotic [18] and $89.8 \%$ with a mac- rolide antibiotic [19], close to the results presented here. Although there may be many reasons for differences between the results in clinical trials and actual real-life effectiveness, the results we found are quite similar.

A second limitation to conclude the observed protective effect of antibiotics is the absence of an experimental design (randomization and double-blind treatment) in this cohort study of real-life medical practice. Considering the large number of participating physicians, one might expect that antibiotics would be more often prescribed in the more severe cases, no antibiotics in the milder cases and quasi-random choice for the patients in between. If anything, this would bias the results towards less apparent effectiveness of antibiotics and therefore an underestimation of this effect. Indeed, such potential channelling may have participated to the lesser effectiveness of antibiotics found here compared with that reported in recent metaanalyses of clinical trials [14-16].

In a recent Cochrane Collaboration meta-analysis, cure or improvement of maxillary sinusitis was found in $90 \%$ of the antibiotic group and $80 \%$ of the placebo group [16]. Individual randomized controlled trials (RCTs) do, however, report a large variation in cure or improvement. In 11 RCTs selected for another recent meta-analysis, cure or improvement ranged from $40 \%$ to $74 \%$ with antibiotics and from $30 \%$ to $66 \%$ with placebo [15]. In 16 RCTs selected for a third meta-analysis of individual patient data, cure ranged from $35 \%$ to $98 \%$ with antibiotics and $28 \%$ to $89 \%$ with placebo [14]. The wide range of RCT results is related to different definitions for cure and improvement, the method employed to obtain data (patients diary, telephone interview, clinical examination, etc.) and a conservative hypothesis in case of missing data. The corresponding odds ratios of antibiotic efficacy in these meta-analyses were $1.52(95 \% \mathrm{Cl} 1.02,2.27)[16], 1.37$ (95\% Cl 1.13, 1.66) [15] and $1.64(95 \% \mathrm{Cl} 1.35,2.00)$ [14].The substantially lower effect of antibiotics found here (RR 1.09, 95\% Cl 1.07, 1.10) can be attributed to the higher rate of AS resolution in the two groups studied. However, despite this difference, the number needed to treat of $13(95 \% \mathrm{Cl} 8,22)$ that we found is close to the 15 reported by Young et al. in their metaanalysis [15]. This shows that the differences between clinical trials and real-life observation may be one of absolute magnitude related to success/failure criteria, but that the relative efficiency of antibiotics seems quite stable.

The protective effect of antibiotics was quite homogeneous among all subgroups studied. However, patients with poor oro-dental condition and patients with previous antibiotic use were clearly more likely to benefit from antibiotic prescription. No other condition or symptom distinguished patients who were more or less likely to benefit from antibiotics. A diagnosis of bacterial infection would be therefore one of the main issues to select patients who might most benefit from antibiotics. The French guidelines [10] failed both to impact on prescribers' decision to use antibiotics and to identify patients with a greater protec- 


\section{B] CP P. Blin et al.}

\section{Table 2}

Uni- and multivariate analysis of factors associated with initial treatment failure (Cox proportional-hazards regression model)

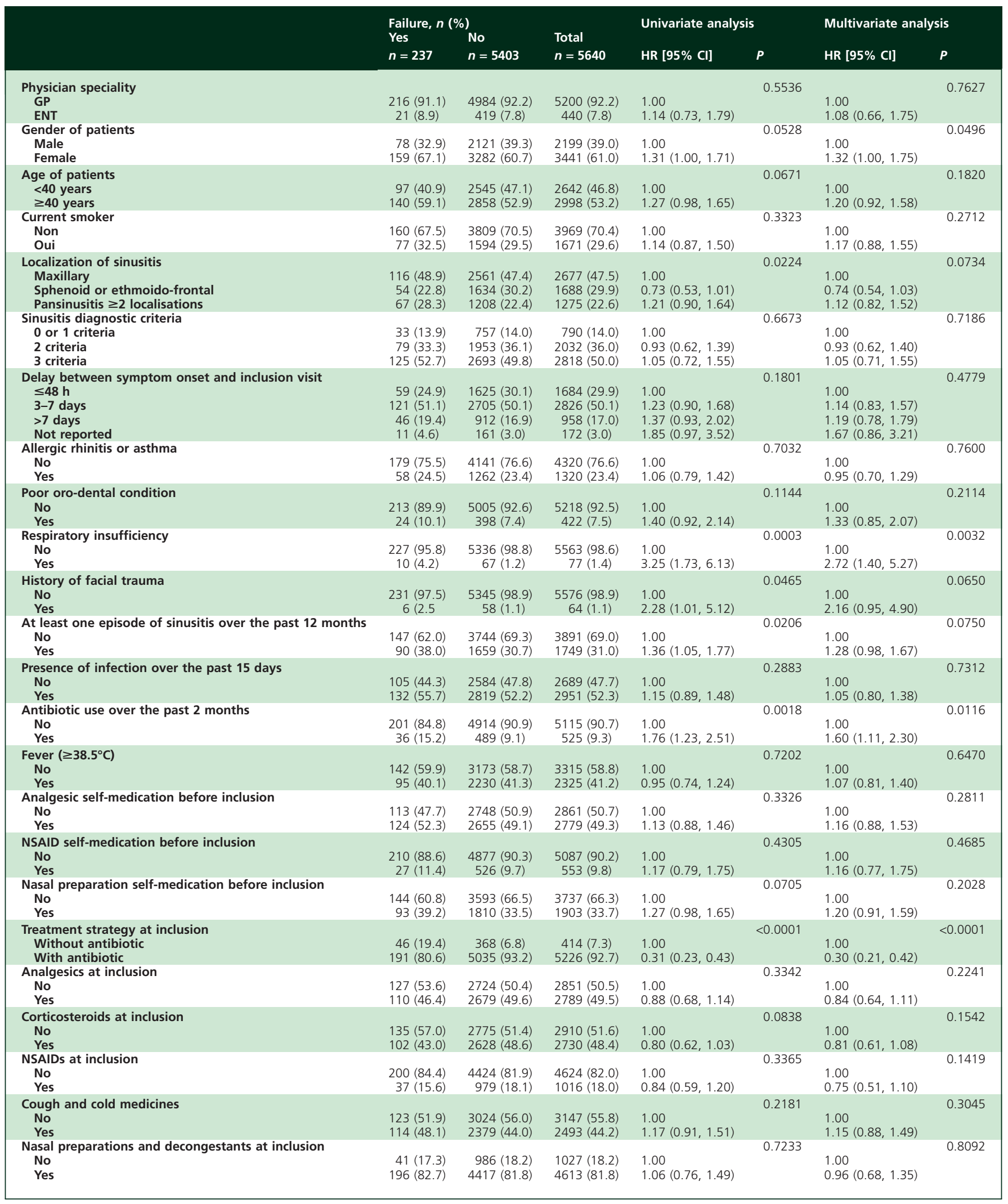




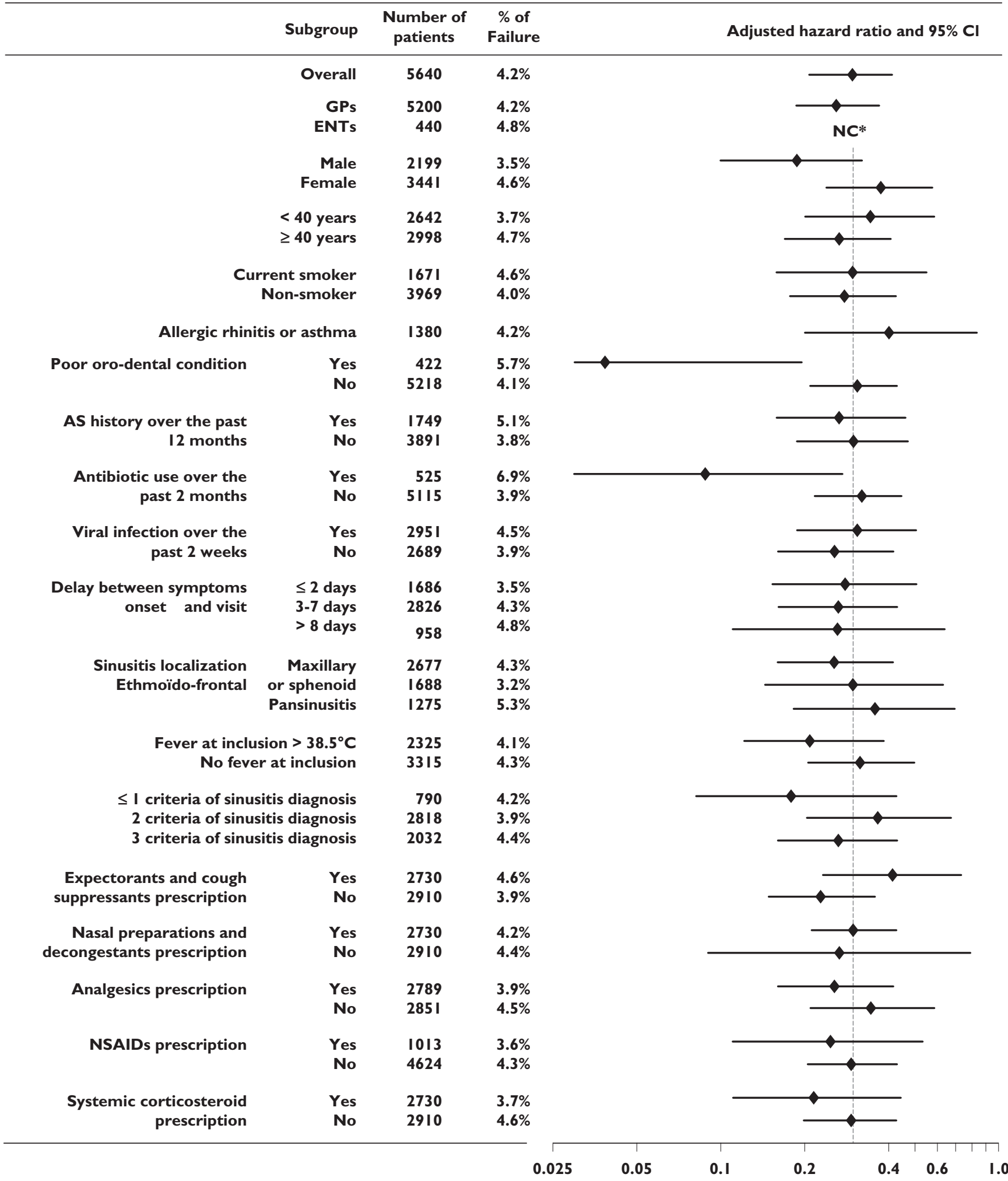

*NC Not calculable (no failure for patient without antibiotic)

\section{Figure 2}

Relative risk of failure of antibiotic prescription for main subgroups 
tive effect of antibiotics. The clinical practice guidelines from the American Academy of Otolaryngology-Head and Neck Surgery Foundation $[2,12]$ argued that ABS can be distinguished from acute viral rhinosinusitis only by the persistence of symptoms (purulent nasal discharge with either nasal obstruction or facial pain-pressure-fullness or both) after 10 days or a worsening of these after an initial improvement during the same period. One might believe that patients treated with antibiotics came to consult because of the failure of non-intervention, but in our study, the protective effect of antibiotics was the same whatever the duration of symptoms before consultation. Fever is considered in the latest clinical practice guidelines from the American Academy of Otolaryngology-Head and Neck Surgery Foundation [2] as a criteria for severe illness to be treated initially with an oral antibiotic, but had no influence on antibiotic efficiency in our study, nor did it predict treatment failure in patients not prescribed antibiotics.

Given that a high proportion of the cases of ABS not prescribed antibiotics resolve spontaneously, US treatment guidelines advocate a policy of 'watchful waiting' combined with symptomatic therapy as an initial management strategy; antibiotic therapy is recommended if symptoms worsen or persist for more than 7 to 10 days [4]. Patients not initially prescribed an antibiotic in the current study could be considered to be treated using an approach similar to the 'watchful waiting' strategy and for most this did not fail. From a financial point of view such an approach is supported by meta-analyses reported by de Bock et al. [22] and Benninger et al. [23]. These, respectively, showed that postponing initiation of the antibiotic treatment for 7 days is the most cost-effective strategy for AS and that overall expenses due to empirical antibiotic prescription to any patient would exceed initial and additional costs in case of treatment failure. However, although most patients with acute purulent sinusitis recover without antibiotic treatment, some could be exposed to the risk of serious complications and sequelae that include meningitis, brain abscess, orbital cellulites and orbital abscess [2]. In the meta-analysis of individual patient data [15], one case of brain abscess was reported among the 1381 patients randomly assigned to placebo. Even if such serious events occurred for one case out of 10000 in the population, 'watchful waiting' could expose the 20 million ABS cases per year in the USA $[12,24]$ to the risk of about 2000 cases of serious events. In our study of almost 6000 cases of AS, most of which received antibiotics, there was no instance of such complications.

The very high rate of antibiotic prescription in this cohort of AS patients is in accordance with that observed in previous European and US studies (72\% to 92\%) [7, 25-27], in spite of the spontaneous cure of most ABS [7], the clear association between increased used of antibiotics and antibiotic resistance [28] and the recommendations of 'watchful waiting strategy' [12]. These studies [7, 25-27] in fact show little use of the 'watchful waiting strategy'. The latest recommendations from the American Academy of Otolaryngology Head and Neck Surgery Foundation [2] consist of an initial 'watchful waiting' strategy for patients with non-severe illness at presentation (mild pain and temperature $<38.3^{\circ} \mathrm{C}$ or $101^{\circ} \mathrm{F}$ ) and oral antibiotics for patients with severe illness (moderate to severe pain or temperature $\geq 38.3^{\circ} \mathrm{C}$ or $101^{\circ} \mathrm{F}$ ). Such an approach seems more applicable and acceptable to physicians and patients in current medical practice and limits the risk of serious complication. From the results presented here, it would also be legitimate to consider the use of antibiotics when patients present with a poor oro-dental condition or previous antibiotic use during the past 2 months.

With regards to choice of the initial antibiotic, international recommendations for the management of AS vary considerably across countries [8], and we found no difference in apparent effectiveness between antibiotics $[2,18]$.

This real-life study confirms that most of the few AS cases not initially prescribed antibiotics resolve [1]. Antibiotics reduce the risk of treatment failure, the duration of symptoms and treatment by symptomatic drugs. None of the usual diagnostic criteria for bacterial sinusitis was effective to select AS that had a better chance of resolving with antibiotics.

Taking into account the benefit for the patient, the risk of complications and the impact of antibiotic use on antibiotic resistance, initial antibiotics treatment in ABS should probably be reserved for patients with initially severe presentation and certainly for patients with the greatest potential benefit: patients with poor oro-dental condition and patients with antibiotic treatment within the previous 2 months. The choice of the actual antibiotic seems completely indifferent to the results and we would recommend the use of the cheapest available.

\section{Competing interest}

Yola Moride has received consulting fees from SanofiAventis Canada to provide advice on pharmacoepidemiology and risk management. She is currently holding funds in the form of an arms-length, unrestricted grant to the University of Montreal for an antidepressant project. Miriam Sturkenboom is running a group that conducts research for various pharmaceutical companies, nothing related to this research. There are no other competing interests to declare.

\section{Exclusive licence statement}

The corresponding author has the right to grant on behalf of all authors and does grant on behalf of all authors, an exclusive licence (or non exclusive for government employees) on a worldwide basis to the British Journal of Clinical Pharmacology. 


\section{Contributors and guarantor(s)}

$P B, S B, S L, R L, H T, C D$ and $N M$ were involved in the study design and implementation of the study and its analysis and interpretation.

$J B, J-L D, P E, J F, Y M, D P, B R, M S$ (independent scientific committee) supervised the study design and execution.

$R L, M-A B, D J$ performed data management and statistical analyses.

PB and NM wrote the first draft of the manuscript and take responsibility for the integrity of the data and the accuracy of the data analysis (guarantors).

All authors had full access to all of the data (including statistical reports and tables) as well as the first draft and subsequent revisions of the manuscript.

NM and PB had full access to all of the data in the study and take responsibility for the integrity of the data and the accuracy of the data analysis.

\section{Ethics approval, funding, funder role, author data access and independence}

The study was performed at the request of the French National Health Authority to improve the understanding of the epidemiology, diagnosis, management and outcome of AS at the time of telithromycin marketing. The study was funded by an unconditional grant from Sanofi-Aventis France who had no direct role in the study design, in the collection, analysis and interpretation of data, in the writing of the manuscript and in the decision to submit the manuscript for publication. A representative from Sanofi-Aventis was an observer on the study's scientific committee, as was a representative from the French regulatory authorities. All authors are independent from the study sponsor.

An independent scientific committee composed of two GPs, two ENT specialists, an infectiologist, a pharmacologist, two epidemiologists and a biostatistician supervised the study. None was part of the study team, or employees of Bordeaux University or Sanofi-Aventis. All contracts and confidentiality agreements for the scientific committee were signed with the University of Bordeaux, which provided compensation for time and travel expenses.

The protocol received the agreement from the national professional ethics committee (Conseil National de l'Ordre des Médecins), the committee in charge of data-protection in biomedical research in France (Comité Consultatif sur le Traitement de l'Information en matière de Recherche dans le domaine de la Santé) and the French National commission for data-protection (Commission Nationale de I'Informatique et des Libertés).

\section{Data sharing}

Data ownership is shared between Sanofi-Aventis and University of Bordeaux. Requests for the technical appendix, statistical code and dataset should be addressed to Patrick.Blin@pharmaco.u-bordeaux2.fr, with a description of the reasons for the request, which will be examined by the Scientific Committee.

\section{STROBE statement}

All the items in the Strobe statement pertaining to cohort studies have been considered and completed or included in this paper.

We thank the physicians who participated in the study as well as Philip Robinson for help in preparation of the manuscript.

\section{REFERENCES}

1 Hwang PH. A 51-year-old woman with acute onset of facial pressure, rhinorrhea, and tooth pain: review of acute rhinosinusitis. JAMA 2009; 301: 1798-807.

2 Rosenfeld RM. Clinical practice guideline on adult sinusitis. Otolaryngol Head Neck Surg 2007; 137: 365-77.

3 Ah-See KW, Evans AS. Sinusitis and its management. BMJ 2007; 334: 358-61.

4 Marple BF, Brunton S, Ferguson BJ. Acute bacterial rhinosinusitis: a review of U.S. treatment guidelines. Otolaryngol Head Neck Surg 2006; 135: 341-8.

5 Lindbaek M. Acute sinusitis - to treat or not to treat? JAMA 2007; 298: 2543-4.

6 Lindbaek M. Acute sinusitis: guide to selection of antibacterial therapy. Drugs 2004; 64: 805-19.

7 Desrosiers M, Klossek JM, Benninger M. Management of acute bacterial rhinosinusitis: current issues and future perspectives. Int J Clin Pract 2006; 60: 190-200.

8 Klossek JM, Federspil P. Update on treatment guidelines for acute bacterial sinusitis. Int J Clin Pract 2005; 59: 230-8.

9 Le Annie V, Simon RA. Making the call: the diagnosis of acute community-acquired bacterial sinusitis. Am J Rhinol 2006; 20: 658-61.

10 Agence Française de Sécurité Sanitaire des Produits de Santé. Systemic antibiotic treatment in upper and lower respiratory tract infections: official French guidelines. Clin Microbiol Infect 2003; 9: 1162-78.

11 Groupe d'Etude des Maladies Infectieuses. Current approaches to community-acquired acute maxillary rhinosinusitis or sinusitis in France and literature review. Rhinol Suppl 2001; (17): 1-38.

12 Anon JB, Jacobs MR, Poole MD, Ambrose PG, Benninger MS, Hadley JA, Craig WA. Antimicrobial treatment guidelines for acute bacterial rhinosinusitis. Otolaryngol Head Neck Surg 2004; 130: 1-45. 
13 Gwaltney JM Jr. Acute community-acquired sinusitis. Clin Infect Dis 1996; 23: 1209-23.

14 Falagas ME, Giannopoulou KP, Vardakas KZ, Dimopoulos G, Karageorgopoulos DE. Comparison of antibiotics with placebo for treatment of acute sinusitis: a meta-analysis of randomised controlled trials. Lancet Infect Dis 2008; 8: 543-52.

15 Young J, De Sutter A, Merenstein D, van Essen GA, Kaiser L, Varonen $\mathrm{H}$, Williamson I, Bucher HC. Antibiotics for adults with clinically diagnosed acute rhinosinusitis: a meta-analysis of individual patient data. Lancet 2008; 371 : 908-14.

16 Ahovuo-Saloranta A, Borisenko OV, Kovanen N, Varonen $\mathrm{H}$, Rautakorpi UM, Williams JW Jr, Makela M. Antibiotics for acute maxillary sinusitis. Cochrane Database Syst Rev 2008; (2): CD000243.

17 Marple BF. Editorial commentary: dilemma in trial design: do current study designs adequately evaluate effectiveness antibiotic in ABRS? Otolaryngol Head Neck Surg 2005; 133 : 200-1.

18 Piccirillo JF, Mager DE, Frisse ME, Brophy RH, Goggin A. Impact of first-line vs second-line antibiotics for the treatment of acute uncomplicated sinusitis. JAMA 2001; 286: 1849-56.

$19 \mathrm{Wu} J \mathrm{H}$, Howard DH, McGowan JE Jr, Frau LM, Dai WS. Patterns of health care resource utilization after macrolide treatment failure: results from a large, population-based cohort with acute sinusitis, acute bronchitis, and community-acquired pneumonia. Clin Ther 2004; 26: 2153-62.

20 Sicart D. Les médecins, Estimations au 1er janvier 2004. Drees, Série statistiques n78, 2005.
21 Blin P, Olie JP, Sechter D, Petitjean F, Cialdella P, Gerard A, Hanssens L, Westerloppe J. [Neuroleptic drug utilization among schizophrenic outpatients]. Rev Epidemiol Sante Publique 2005; 53: 601-13.

22 de Bock GH, van Erkel AR, Springer MP, Kievit J. Antibiotic prescription for acute sinusitis in otherwise healthy adults. Clinical cure in relation to costs. Scand J Prim Health Care 2001; 19: 58-63.

23 Benninger MS, Sedory Holzer SE, Lau J. Diagnosis and treatment of uncomplicated acute bacterial rhinosinusitis: summary of the Agency for Health Care Policy and Research evidence-based report. Otolaryngol Head Neck Surg 2000; 122: 1-7.

24 McCaig LF, Hughes JM. Trends in antimicrobial drug prescribing among office-based physicians in the United States. JAMA 1995; 273: 214-9.

25 Ashworth M, Charlton J, Ballard K, Latinovic R, Gulliford M. Variations in antibiotic prescribing and consultation rates for acute respiratory infection in UK general practices 1995-2000. Br J Gen Pract 2005; 55: 603-8.

26 Kuyvenhoven M, van Essen G, Schellevis F, Verheij T. Management of upper respiratory tract infections in Dutch general practice; antibiotic prescribing rates and incidences in 1987 and 2001. Fam Pract 2006; 23: 175-9.

27 Varonen H, Rautakorpi UM, Huikko S, Honkanen PO, Klaukka T, Laippala P, Palva E, Roine R, Sarkkinen H, Makela M, Huovinen P. Management of acute maxillary sinusitis in Finnish primary care. Results from the nationwide MIKSTRA study. Scand J Prim Health Care 2004; 22: 122-7.

28 Goossens H, Grabein B. Prevalence and antimicrobial susceptibility data for extended-spectrum beta-lactamaseand AmpC-producing Enterobacteriaceae from the MYSTIC Program in Europe and the United States (1997-2004). Diagn Microbiol Infect Dis 2005; 53: 257-64. 